OPEN ACCESS

Edited by:

Rui F. Oliveira,

University Institute of Psychological,

Social and Life Sciences

(ISPA), Portugal

Reviewed by:

David Goncalves,

University of Saint Joseph, Macau

Ethan K. Scott,

The University of

Queensland, Australia

*Correspondence:

Summer B. Thyme

sthyme@gmail.com

Specialty section

This article was submitted to Individual and Social Behaviors,

a section of the journal

Frontiers in Behavioral Neuroscience

Received: 15 September 2020

Accepted: 10 December 2020

Published: 18 January 2021

Citation:

Joo W, Vivian MD, Graham BJ,

Soucy ER and Thyme SB (2021) A

Customizable Low-Cost System for

Massively Parallel Zebrafish Behavioral

Phenotyping.

Front. Behav. Neurosci. 14:606900

doi: 10.3389/fnbeh.2020.606900

\section{A Customizable Low-Cost System for Massively Parallel Zebrafish Behavioral Phenotyping}

\author{
William Joo ${ }^{1}$, Michael D. Vivian ${ }^{2}$, Brett J. Graham ${ }^{3}$, Edward R. Soucy ${ }^{3}$ and \\ Summer B. Thyme ${ }^{2 *}$
}

${ }^{1}$ Biozentrum, University of Basel, Basel, Switzerland, ${ }^{2}$ Department of Neurobiology, University of Alabama at Birmingham, Birmingham, AL, United States, ${ }^{3}$ Center for Brain Science, Harvard University, Cambridge, MA, United States

High-throughput behavioral phenotyping is critical to genetic or chemical screening approaches. Zebrafish larvae are amenable to high-throughput behavioral screening because of their rapid development, small size, and conserved vertebrate brain architecture. Existing commercial behavioral phenotyping systems are expensive and not easily modified for new assays. Here, we describe a modular, highly adaptable, and lowcost system. Along with detailed assembly and operation instructions, we provide data acquisition software and a robust, parallel analysis pipeline. We validate our approach by analyzing stimulus response profiles in larval zebrafish, confirming prepulse inhibition phenotypes of two previously isolated mutants, and highlighting best practices for growing larvae prior to behavioral testing. Our new design thus allows rapid construction and streamlined operation of many large-scale behavioral setups with minimal resources and fabrication expertise, with broad applications to other aquatic organisms.

Keywords: zebrafish, high-throughput screens, automated behavior, pre-pulse inhibition, neuropsychiatric disease, high-speed tracking, DanioVision, ZebraBox

\section{INTRODUCTION}

High-throughput behavioral tracking offers great potential for large-scale mutant phenotyping (Thyme et al., 2019) and drug screening (MacRae and Peterson, 2015). Indeed, drug screens have revealed conserved signaling pathways that regulate complex behaviors in both zebrafish and mammals (Kokel et al., 2010; Rihel et al., 2010; Leung and Mourrain, 2016). Furthermore, larval zebrafish maintained in 96-well plate format execute diverse behaviors including prepulse inhibition (Burgess and Granato, 2007), sleep (Chiu et al., 2016), seizures (Griffin et al., 2020), prey consumption (Jordi et al., 2018), and responses to visual (Randlett et al., 2019), acoustic (Wolman et al., 2011), or thermal stimuli (Chiu et al., 2016). Many researchers use commercial systems to test these behaviors, but such solutions are limited in their adaptability and prohibitively costly when many parallel systems are required.

For example, two of the most commonly used commercial systems are the DanioVision from Noldus and the ZebraBox from ViewPoint. Without add-ons, these systems provide only baseline movement tracking (i.e., activity, swimming bursts, thigmotaxis) and LED light control. While add-ons such as high-speed cameras, acoustic stimulation, and temperature control are available, they greatly increase system cost. Furthermore, users are often limited to commercially provided analysis code and data processing formats. 
To bypass these challenges, we present building plans for a modular behavioral testing setup (Figure 1A), together with software for data acquisition and analysis. Our new design significantly extends systems previously validated in a large-scale mutant screen (Thyme et al., 2019), with more precise control over a broader range of assays and greater ease of construction. This system includes most of the assays of commercially available solutions and easily accommodates additional modules. Our analysis software utilizes a high-performance computing cluster for parallel processing of multi-day datasets with hundreds of user-defined events. Additionally, we outline best experimental practices for yielding consistent and reliable behavioral data. This fully customizable and modular setup can be easily adapted as new behavioral assays are published, significantly lowering barriers to large-scale phenotyping approaches.

\section{MATERIALS AND METHODS}

\section{Materials}

All components and costs are described in Supplementary Material Table 1, and all schematics are included in FabricationFiles (Supplementary Material Data Sheet 2). While access to a laser cutter and 3D printer substantially decreases cost and time of construction, online manufacturing websites can easily produce equivalent parts (see Supplementary Material Data Sheet 1).

\section{Box Assembly}

Supplementary Material describes all assembly steps. The setup housing consists of a light-insulated enclosure, a camera to track fish motion, and a computer/electronics setup to deliver stimuli (Figure 1A). The enclosure was laser-cut from high-density polyethylene (HDPE) and fastened with 80/20 rails (Figure 1B).

The enclosure contains a white LED panel to deliver ambient light or stimuli, an infrared (IR) light to visualize animals, and a 3D-printed fish plate holder with a mounted acoustic transducer (Figure 1B). The white LED panel is mounted on an acrylic shelf and illuminates fish from below, while the IR light rests behind and reflects off the white light panel. Fish were detected with a Grasshopper3 camera (FLIR Systems) and a $50 \mathrm{~mm}$ fixed focal length lens with an IR filter.

\section{Data Acquisition}

See Supplementary Material (Data Sheet 1) for detailed operation instructions.

\section{Computer Hardware}

The setup was operated using a standard desktop computer and custom LabVIEW software (Supplementary Material Data Sheet 3). Minimum hardware requirements for the most computationally demanding assay (acoustic habituation; 1-s movies at 285 frames-per-second [fps] acquired every 2s) were 16.0 GB RAM, an Intel Core i7-9700 processor, Windows 10 , and a 1 TB Solid-State Drive. For those who do not have access to a full LabVIEW license, a compiled executable can be provided upon request. Running the executable will require a nominal license fee for the National Instruments Vision Development Tools package.

\section{Stimulus Delivery and Data Collection}

Acoustic and visual stimuli were controlled by a circuit board that communicates between LabVIEW software and system devices (Figure 2A). A Teensy 3.6 microcontroller and custom Arduino script relays stimulus command strings to LabVIEW (Figure 2B). Each "command string" specifies stimulus parameters such as amplitude (a), frequency (f), duration (d), and delay times (D) (full list in Supplementary Material Data Sheet 1). The microcontroller then sends voltage changes to the surface transducer or LED light panel to produce stimuli. The "command ID” (Figure 2B) specifies the LabVIEW event type, such as highspeed movie acquisition during stimulus presentation.

To run an experiment, users (1) construct an events file (Supplementary Material Data Sheet 3) with desired command strings, (2) designate regions of interest (ROIs) using a separate LabVIEW script (Generate ROIs.vi) (Figure 2C), which generates an ROI binary data file (rois) and ROI string text file (rois_string) (Supplementary Material Data Sheet 3). ROIs can match many different multi-well plate formats. (3) Select the events file, the ROI binary data file, and a png image of the plate using the LabVIEW graphical user interface (GUI) (Figure 2D). Users also define data output names and folders. See Supplementary Material Data Sheet 1 for detailed setup instructions. The ROI string text file is used in later stages of the analysis.

$30 \mathrm{fps}$ data is collected for the duration of the experiment in two formats: the change in pixels between each frame within each ROI, and the coordinates of the centroid of each fish in each ROI (Slow-speed data, Figure 2E). User-defined LabVIEW events trigger acquisition of one-second movies at $285 \mathrm{fps}$ (highspeed data). LabVIEW can also trigger acquisition of $30 \mathrm{fps}$ movies of desired length.

\section{Data Analysis Software}

Our analysis pipeline (Figure 3) is based in the Python programming language. All analyses were performed on a highperformance computing (HPC) cluster due to vastly increased parallel processing capacity. LabVIEW generates slow-speed motion (delta pixels) and centroid (coordinates) data, while our Python scripts extract motion and centroid from high-speed data (Figure 3A). As in LabVIEW, a centroid for each fish is identified in each ROI to determine coordinates. Our typical behavior run produces close to one thousand high-speed movies (784 in example run), making parallel processing advantageous at this tracking step, particularly if comparing multiple genotypes or analyzing data from multiple experiments. However, all scripts are compatible with local analysis on a single core and can be adapted to parallel processing environments other than HPC. Tracking of 784 movies takes $<1.5 \mathrm{~h}$ on a standard iMac (vs. $<5$ min on the HPC cluster). Anaconda3 and OpenCV must be installed. Scripts for analysis of the tracked data have been extensively profiled for efficient analysis of 3 days of movement data and hundreds of high-speed movies. Each analysis run takes between 1.5 and $3.5 \mathrm{~h}$ on a single core, depending on the number 

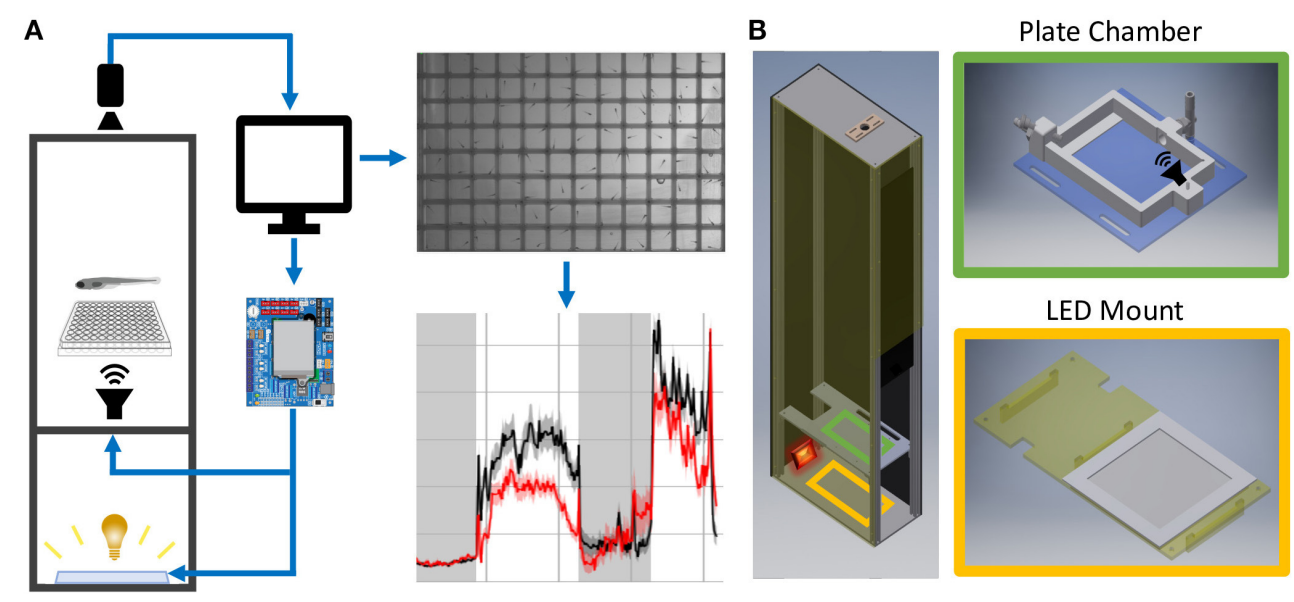

FIGURE 1 | Behavior Box Overview. (A) Schematic of the behavior box setup. A high-speed camera is mounted on top of the box and focused on the fish plate. A microcontroller circuit is connected to a white LED panel at the bottom of a box and a surface transducer attached to the plate holder, which deliver visual and acoustic stimuli, respectively. The microcontroller and camera are connected to a desktop computer, which uses custom LabVIEW software for data acquisition and experiment control. (B) Left: Setup enclosure is affixed to an aluminum frame with clear acrylic shelving for the plate holder and LED panel. Right: The 3D printed fish chamber includes input/output nozzles for water circulation and a screw stud for the surface transducer. See supplemental files in Supplementary Material Table 1 for parts list and assembly instructions (Data Sheet 1: Supplementary Figures 1-9).

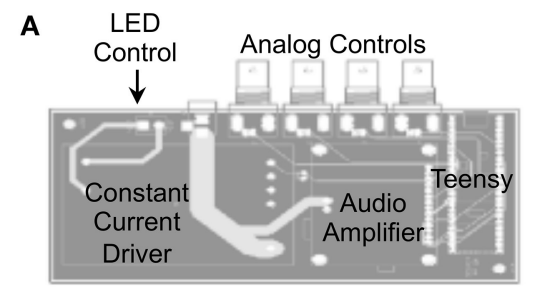

D

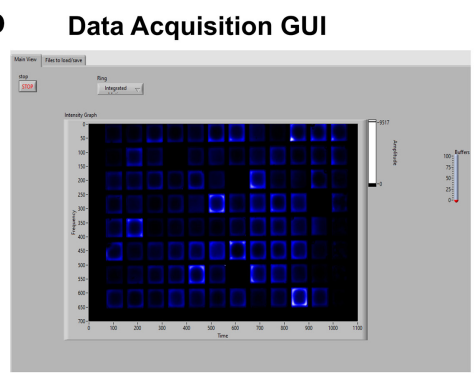

B

Event Specification

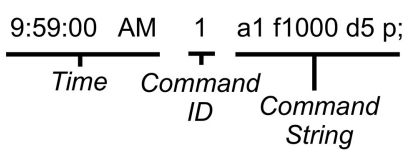

E

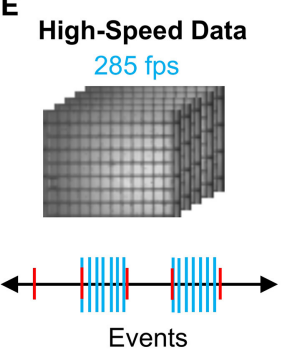

C
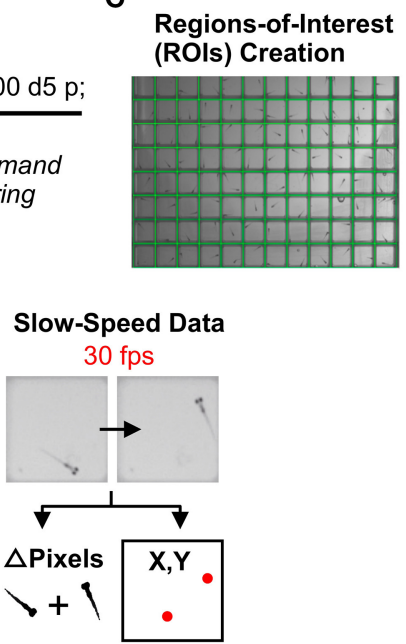

FIGURE 2 | Data Acquisition Control. (A) Printed circuit board for electronics control. The LED light panel and surface transducer are manipulated by a Teensy 3.6 microcontroller with a constant current LED driver and an audio amplifier. A custom Arduino script with command options is uploaded to the microcontroller. The board also includes four BNC connectors wired to GPIO pins on the Teensy that support digital input/output, analog input, and other functionality configurable from software. For instance, a photodiode can be connected to calibrate the light panel. (B) Example command string to specify an event such as lights-off or high-speed movie acquisition during stimulus. See Supplementary Software in Supplementary Material Data Sheet $\mathbf{3}$ for an example events file (eventsfile). (C) Users define regions of interest corresponding to each well using a LabVIEW graphical interface. Event parameters and ROls are then transferred to main experiment software. (D) The LabVIEW data acquisition interface can display fish movements in real-time. (E) High-speed data is captured as 1-s 285 fps AVI movies as specified in the events file. Slow-speed data is collected at $30 \mathrm{fps}$ to produce motion (delta pixels) and centroid (coordinates of fish centroid) files for the entirety of the experiment. Slow-speed data is continuously acquired regardless of high-speed events. See Supplementary Figures 10-15 (Data Sheet 1) for information regarding data acquisition pipeline.

of animals. The total size of a run is $\sim 20 \mathrm{~GB}$ prior to analysis, which expands to $60-120 \mathrm{~GB}$ depending on the number of groups compared, due to the thousands of graphs generated. Storage needs can be reduced by producing or saving graphs only for measures with statistically significant differences.

Input data from slow- and high-speed tracking is processed to generate numerous measurements and output graphs (Figure 3B) ranging from classic behaviors such as sleep bouts and waking activity (Chen et al., 2017) to recently published observations such as turn angle preference during dark flash response (Horstick et al., 2020) (Python/examplefiles/BehavioralMetrics.xlsx and Python/PlotParameters) (Supplementary Material Data Sheet 3). A Fish object is created for each animal and contains all 


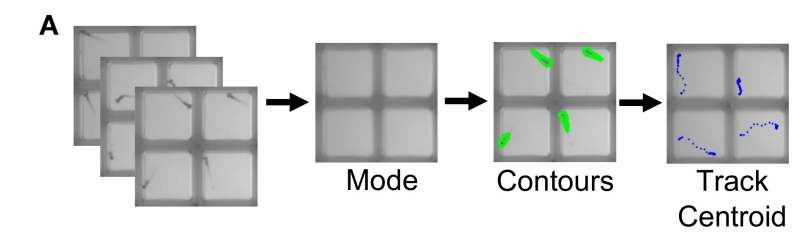

B

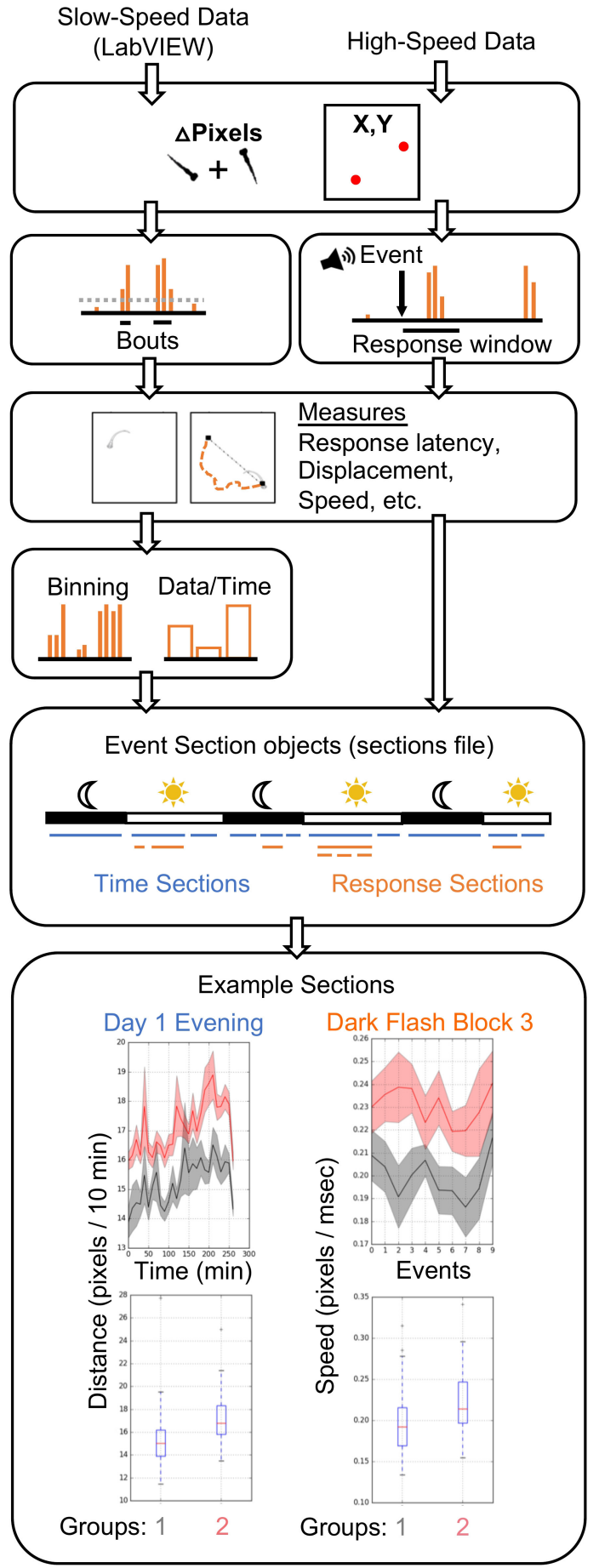

FIGURE 3 | Data Analysis Pipeline. (A) The high-speed movies are analyzed by calculating and subtracting a mode image to define frame-by-frame fish contours in each well or ROI, and tracking the centroid of each fish to generate

(Continued)
FIGURE 3 | delta pixel and position data. (B) Overview of high- and slow-speed data processing and group comparisons. Fish objects contain all metrics for each fish as well as its genotype (gray = group 1, red = group 2). For slow-speed data, bouts are identified using the delta pixel and positional information. Thresholds are set depending on input data type. Stimulus responses are identified analogously, but are identified with high-speed movie frames. Movement and response features are then calculated, binned if slow-speed data, and plotted based on user defined event sections. For example, slow-speed data is processed in sections based on time, such as "Day 1 Evening" or "Day 2 Night." High-speed data processing considers only the high-speed movie information in a given section, such as the 10 dark flashes in "Dark Flash Block 3." Sections can be overlapping. Current outputs include both a ribbon plot and a box plot for each metric.

associated slow- and high-speed data as well as genotype. Slowspeed data is converted into movement bouts calculated from both motion data and centroid data. Metrics such as frequency, velocity, and fraction of time in well-center are calculated for each bout and binned based on time (such as average velocity / $10 \mathrm{~min}$ ). High-speed data is processed based on the type of event and the parameters of the event string. Identification of an event response depends on modality (visual, acoustic) and the time delays in the string. Metrics analogous to bout properties are then calculated for the response. High-speed and binned slow-speed data are returned to the Fish object as ProcessedData objects, which are then used to generate graphs according to user-defined event sections. The event sections are specified in the sections file, which segments the behavior run into time windows for different assays. For example, an acoustic habituation assay would be analyzed separately from the prepulse inhibition assay. Event sections may also correspond to different times of the run such as night or day, and need not include high-speed events. Sections without high-speed events are referred to as "time" sections. An example sections file is included in the Supplementary Software (Python/sectionsfile) in Supplementary Material Data Sheet 3. Data and statistics are saved and a graph is generated for every combination of an EventSections object and ProcessedData object. A KruskalWallis one-way ANOVA is calculated for every metric, and a linear mixed model (Thyme et al., 2019) is also calculated for baseline data with a time component. The code is also available on GitHub (https://github.com/sthyme/ZebrafishBehavior) and will be updated as improvements are made.

\section{Assays}

The most common multi-well larval zebrafish assays are based on acoustic and visual stimulation, utilizing the surface transducer and the LED light panel. These include responses to increased light or decreased light (dark flash), dark flash habituation, acoustic responses and thresholds, prepulse inhibition, and acoustic habituation. Our setup can test responses to a broad range of acoustic (tones of varying frequency, duration, waveform, and amplitude delivered by surface transducer), visual (whole-field luminance changes such as dark or light flashes), and thermal stimuli (cooling or heating with a water circulation system; see Supplementary Material Data Sheet $\mathbf{1}$ ), and can be further modified for additional assays. The design also 


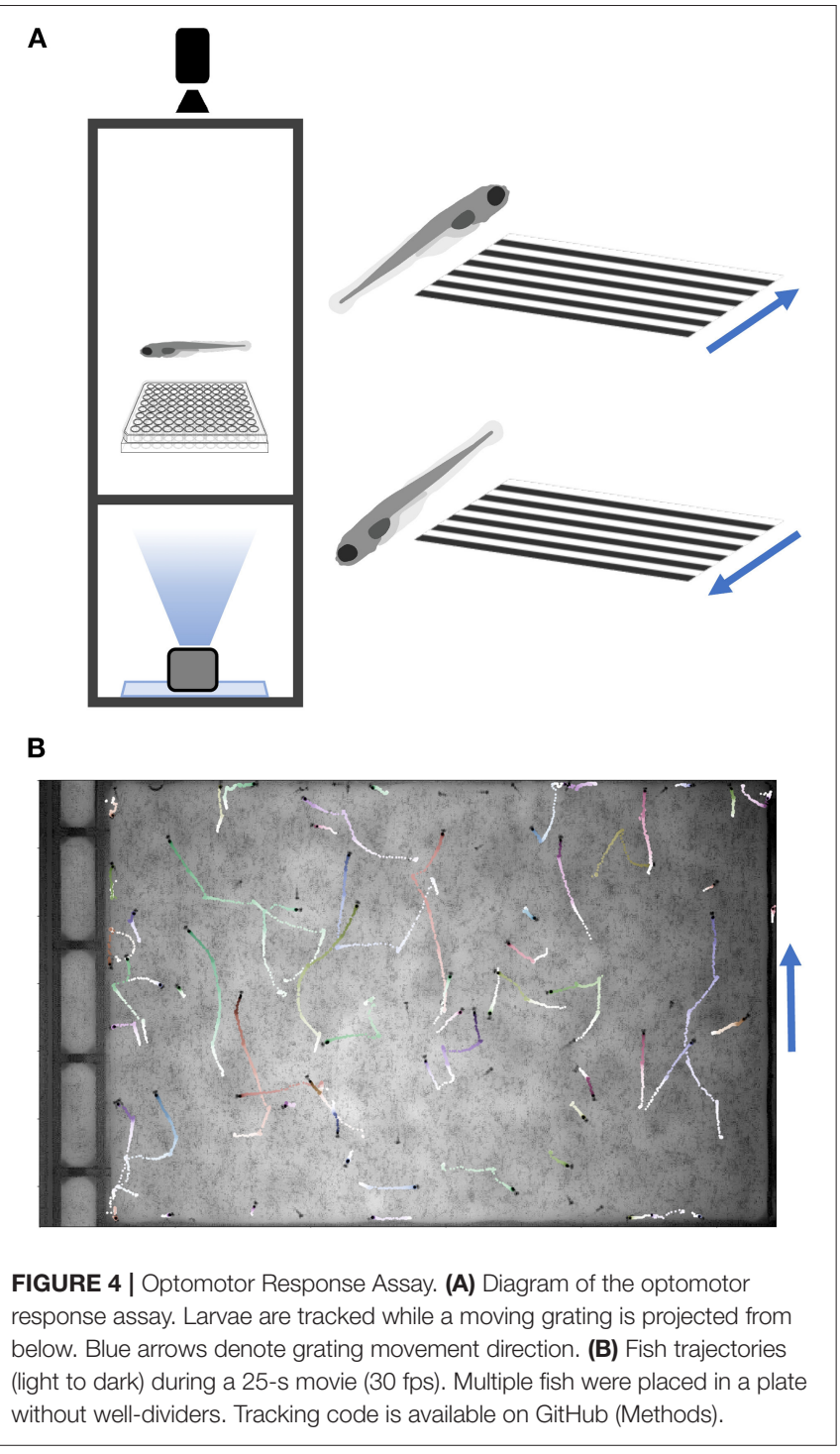

includes a mini-projector underneath the fish plate, which can present user-defined movies such as moving gratings to induce the optomotor response (Figure 4) (Naumann et al., 2016). Movies are presented through LabVIEW via the VLC media player (using movie file paths instead of command strings). Supplementary Software (Python/OMR) in Supplementary Material Data Sheet 3 includes Python scripts to generate gratings and example movies. Code to track multiple animals was completed with a custom script (http://github.com/ docviv/behavior-scripts) based on an algorithm adapted from (Bolton et al., 2019).

To test arousal threshold (Figure 5), we delivered acoustic $(20 \mathrm{~ms}, 625 \mathrm{~Hz}$, square wave-form) or dark flash (1 s) visual stimuli at 12 different intensities in separate experiments: acoustic $=\mathrm{a} 0.0005, \mathrm{a} 0.001, \mathrm{a} 0.003, \mathrm{a} 0.0075, \mathrm{a} 0.01, \mathrm{a} 0.03, \mathrm{a} 0.06$, $\mathrm{a} 0.075, \mathrm{a} 0.1, \mathrm{a} 0.3, \mathrm{a} 0.5, \mathrm{a} 1$, visual $=\mathrm{b} 245, \mathrm{~b} 240, \mathrm{~b} 230, \mathrm{~b} 220$, b210, b200, b175, b150, b125, b100, b50, b0, with baseline light $=\mathrm{b} 250$. Supplementary Figure 16 (Data Sheet $\mathbf{1}$ ) summarizes

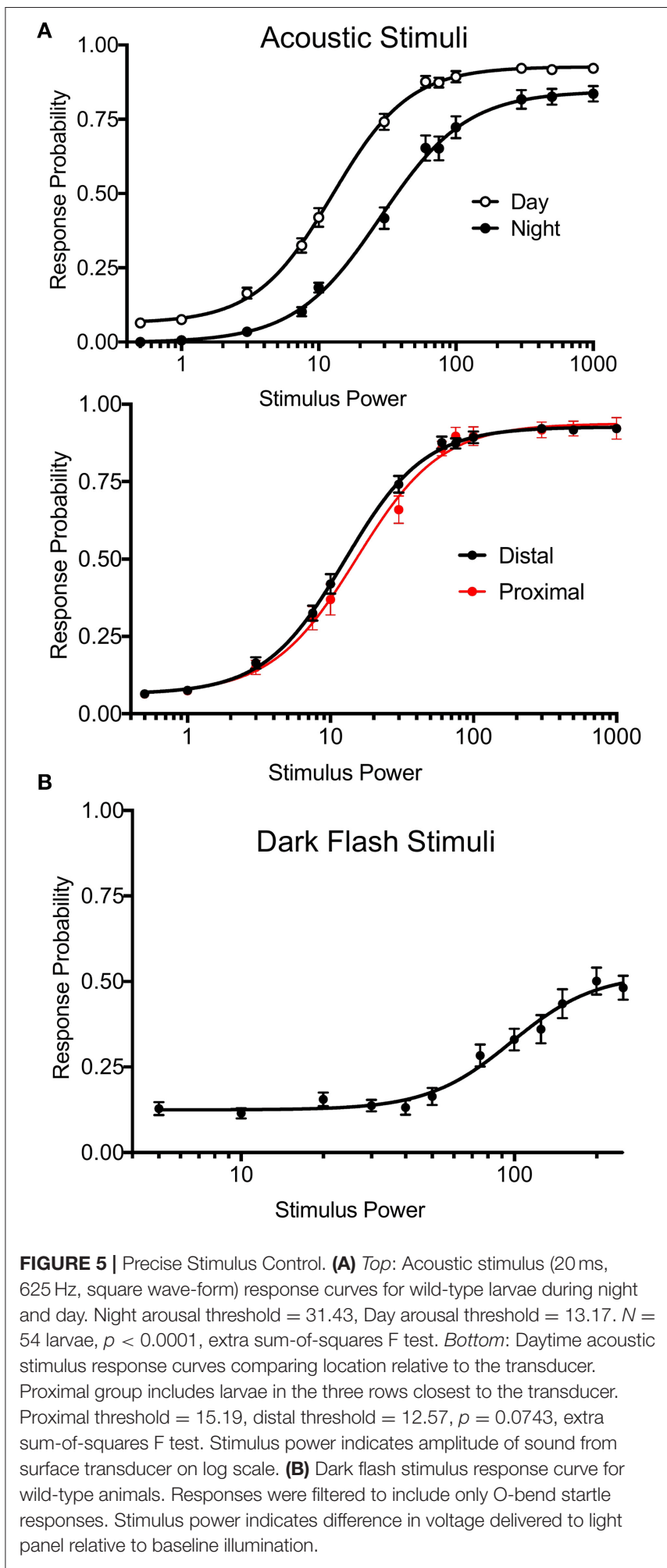

the corresponding decibel and lux values. 30-50 total trials of each intensity were administered in randomized order at $2 \mathrm{~min}$ intervals. Other experiments for mutant and wild-type animals correspond to the event strings in the supplemental events file in Supplementary Material. Stimulus responses were classified 
based on threshold values for movement distance (0.9 pixels), pixel change (3.0), and the number of frames for the distance and the delta pixel measures (respectively, 2 and 3). Users can adjust these parameters via input options in processmotiondata.py (Supplementary Material Data Sheet 3). We also separated bulk responses with movement filters to classify putative C-bends (acoustic startle response) and O-bends (dark flash response). For C-bends, we filtered based on cumulative delta pixels and velocity of the response, as previous studies indicate that short-latency escapes have higher velocity than other escape-like responses (Burgess and Granato, 2007). For O-bends, we filtered based on response time and the sum of heading angles in the response, based on previous studies measuring bend amplitude (Randlett et al., 2019). The O-bend filter was validated using dark flash data in Figure 5, where O-bends should not occur in the absence of stimulus.

\section{Zebrafish Husbandry}

All zebrafish were housed in the Zebrafish Research Facility of the University of Alabama at Birmingham and experiments were approved under protocol number IACUC-21744 (UAB Institutional Animal Care and Use Committee; Birmingham, Alabama). All crosses were derived from a single parental pair (mainly Ekkwill strain) to minimize genetic background differences. Arousal threshold assays were conducted in a mixed $\mathrm{TL} / \mathrm{AB}$ background. Larvae were grown in $150 \times 15 \mathrm{~mm}$ petri dishes with standard methylene blue water, at a density of $<150$ fish per plate. Animals were maintained at $28^{\circ} \mathrm{C}$ and a $14 \mathrm{~h} / 10 \mathrm{~h}$ light/dark cycle. Behavioral experiments were conducted on the same light/dark cycle. Dead material and debris were removed twice before 4 days post-fertilization ( $\mathrm{dpf}$ ) (afternoons of day 0 and day 2). All behavioral assays were conducted on zebrafish larvae 4-7 dpf. Zebrafish of any age can be monitored in this setup with an appropriate holding chamber.

\section{Zebrafish Sample Processing}

Only healthy larvae with normal swim bladder morphology were included in experiments. Larvae were arrayed in 96well plates (E\&K Scientific Cat\#2074, $0.7 \mathrm{~mL} /$ square well volume) in standard methylene blue water. The plate was placed in an ice-water bath until movement abated and sealed with an oxygen-permeable film (Thermo Fisher Scientific Cat\#4311971) to eliminate water evaporation during multiday experiments (Data Sheet 1: Supplementary Figures 17, 18). Sealing is essential to long-term $(>16 \mathrm{~h})$ experiments but incompatible with drug delivery. Accordingly, previous drug screens for sleep modulators periodically refilled evaporated water (Rihel et al., 2010). Sealed plates were placed into the behavior box and secured tightly (screw in one corner) to prevent movement due to the surface transducer. A minimum time of $1 \mathrm{~h}$ between plate sealing and conducting assays is recommended, to allow the larvae to recover from the cooling step and habituate to the environment. Our typical assay includes a $5-7 \mathrm{~h}$ break, as we load the larvae into the boxes on the afternoon of $4 \mathrm{dpf}$ and analyze data starting at $11 \mathrm{PM}$. Temperature inside the setup ranged from 29.5 to $30.5^{\circ} \mathrm{C}$ (measured with a wireless Temp Stick), while room temperature was maintained at $23^{\circ} \mathrm{C}$. For mutant experiments, larvae were genotyped by (1) noting all dead or unhealthy animals, (2) cooling plate on ice until movement ceased, (3) removing water in wells, (4) immersing in sodium hydroxide and transferring to a PCR plate for DNA extraction and amplification.

\section{RESULTS}

\section{Precise Stimulus Control}

Previous versions of our setup used two solenoid tappers and a custom white LED array to deliver acoustic and visual stimuli, respectively (Thyme et al., 2019). Stimulus intensity was inconsistent across setups due to variable construction. For example, solenoid tappers delivered limited and inconsistent tap strengths due to variable height alignment and spring properties, and suffered from artifacts such as inadvertent double or triple tapping (data not shown). The single mounted surface transducer now allows consistent and fine control over a broad range of stimulus durations, voltages, waveforms, and frequencies. Likewise, the new white LED panels deliver consistent luminance across a broad range across setups. We include a simple protocol to calibrate and standardize light levels using a photodiode (see Supplementary Material Data Sheet 1). To validate these modifications, we monitored larval zebrafish responses to acoustic and dark flash stimuli of variable intensities during day and night. By calculating "dose-response" curves for each type of stimulus, we determined arousal threshold, defined as stimulus strength generating half-maximal response probability (Figure 5). Larvae exhibited significantly higher arousal threshold during night relative to day (Figure 5A, top; night threshold $=31.43$, day threshold $=13.17$ ). Acoustic stimulus response probabilities did not differ between fish positioned proximally or distally to the transducer, indicating consistent stimulus delivery across the 96-well plate (Figure 5A, bottom; proximal threshold $=15.19$, distal threshold $=12.57$ ). While maximal dark flash responses matched previously reported levels (Figure 5B) (Woods et al., 2014), we observed improved maximal responses to acoustic stimuli relative to previous assays using solenoids (Lee et al., 2017; Singh et al., 2017). Our modifications thus accommodate previously challenging assays and offer improved standardization.

\section{Mutant Prepulse Inhibition Phenotypes}

We previously demonstrated (Thyme et al., 2019) that mutants for the schizophrenia risk genes atxn7 and sbno1 (Schizophrenia Working Group of the Psychiatric Genomics Consortium, 2014; Girard et al., 2015) exhibit defects in prepulse inhibition (PPI), a sensory-motor gating phenomenon in which a weak prepulse stimulus suppresses an immediately following strong stimulus response. In zebrafish, PPI manifests in response frequency and/or response magnitude. Because previous experiments relied on solenoid tappers, we tested whether the surface transducer recapitulates the PPI assay and phenotypes. Indeed, atxn7 and sbno1 mutants both exhibited decreased PPI relative to sibling controls as previously observed (Figure 6). To further optimize PPI stimulus conditions, we tested multiple frequencies and inter-stimulus intervals (Data Sheet 1: Supplementary Figure 19) and selected 1,000 and $1,400 \mathrm{~Hz}$ for further testing in mutants, for which randomly 


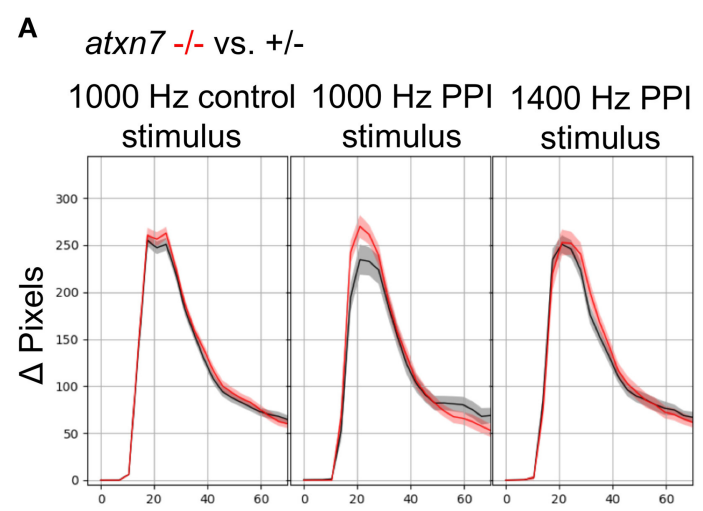

C

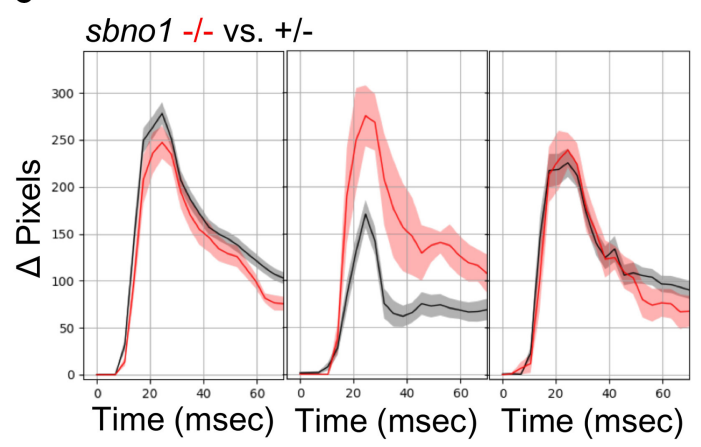

B

atxn7 -/- vs. +/-

$1000 \mathrm{~Hz}$ control $1000 \mathrm{~Hz}$ PPI stimulus stimulus

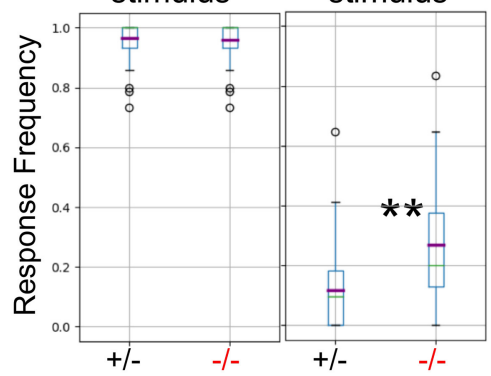

D

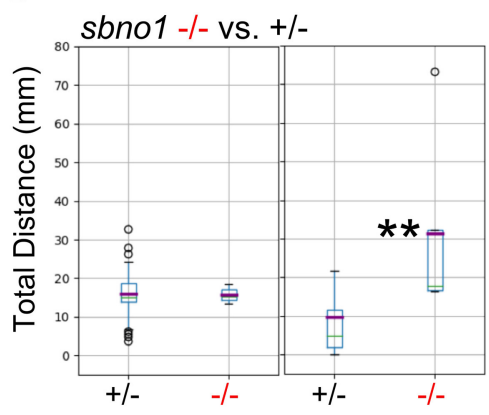

atxn7 -/- vs. +/-

$1000 \mathrm{~Hz}$ control $1000 \mathrm{~Hz}$ PPI
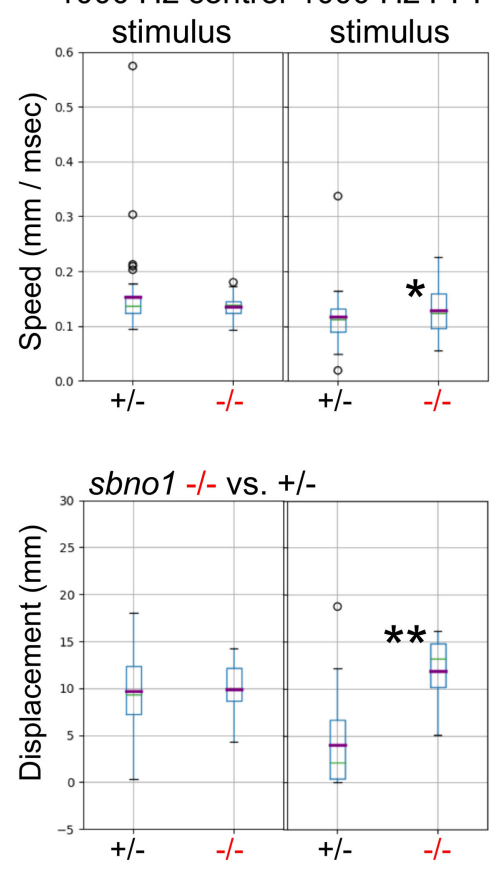

FIGURE 6 | Analysis of Mutants with a Prepulse Inhibition Phenotype. (A) Responses to acoustic prepulse inhibition (PPI) strong stimulus and a control isolated strong stimulus not preceded by a prepulse, quantified as change in pixels during a 1-s high-speed movie. The weak prepulse (not shown) does not elicit a significant response and any prepulse-responding larvae are not considered in the calculations. Red, atxn7 mutant; Gray, sibling control (all 5 dpf). (B) PPI and control escape response frequencies (left) for the atxn7 mutant. $1,000 \mathrm{~Hz}$ PPI Kruskal-Wallis $p$-value $=0.0007,1,000 \mathrm{~Hz}$ Control Kruskal-Wallis $p$-value $=0.95,1,400 \mathrm{~Hz}$ PPI Kruskal-Wallis $p$-value $=0.046$ (not shown). PPI and control response speeds (right): $1,000 \mathrm{~Hz}$ PPI Kruskal-Wallis $p$-value $=0.014,1000 \mathrm{~Hz}$ Control Kruskal-Wallis $p$-value $=0.77,1400 \mathrm{~Hz}$ PPI Kruskal-Wallis $p$-value $=0.66$ (not shown). $N+/-=48,-/-=27$. (C) Responses to acoustic prepulse inhibition (PPI) strong stimulus and a control isolated strong stimulus not preceded by a prepulse, quantified as change in pixels during a 1-s high-speed movie. Red, sbno1 mutant; Gray, sibling control (all 5 dpf). (D) PPI and control escape total response distance moved (left) for the sbno1 mutant. 1,000 Hz PPI Kruskal-Wallis $p$-value $=0.006,1,000 \mathrm{~Hz}$ Control Kruskal-Wallis $p$-value $=0.98,1,400 \mathrm{~Hz}$ PPI Kruskal-Wallis $p$-value $=0.27$ (not shown). PPI and control response displacements (right): $1,000 \mathrm{~Hz}$ PPI Kruskal-Wallis $p$-value $=0.010,1,000 \mathrm{~Hz}$ Control Kruskal-Wallis $p$-value $=0.73,1,400 \mathrm{~Hz}$ PPI Kruskal-Wallis $p$-value $=0.89$ (not shown). $\mathrm{N}+/-=54,-/-=8$. Single asterisk marks $p$-value $<0.05$, double marks $p$-value $<0.01$.

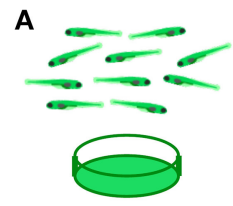

Split 1

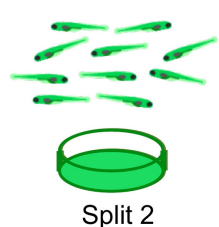

Split 2

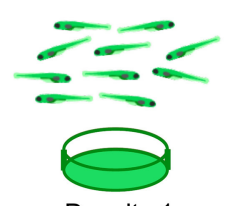

Density 1

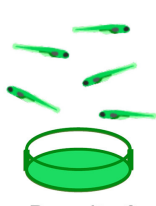

Density 2

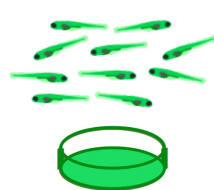

Clutch 1

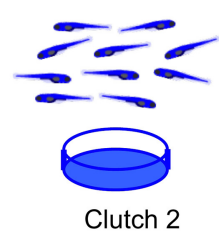

Clutch 2
B

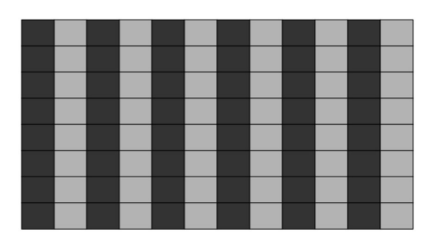

Group 1 and Group 2 Comparison

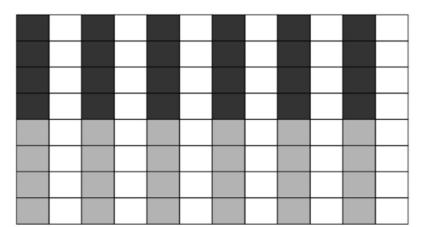

Group 1 Control Comparison

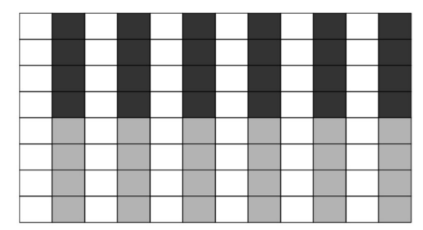

Group 2 Control Comparison

FIGURE 7 | Experimental Design for Wild Type Comparisons. (A) Left: Comparison of a single clutch split between two petri dishes with a density of 140-150 fish per dish. Middle: Comparison of a single clutch split between two petri dishes of different densities: 140-150 fish per dish vs. 60-70 fish. Right: Comparison of two different clutches with equal densities 140-150 fish per dish). (B) Format of the 96-well plate organization for each comparison, where gray and black indicate the two experimental groups loaded in alternating columns. Left: Comparison between the two experimental groups; Middle and Right: control comparisons within each experimental group. 


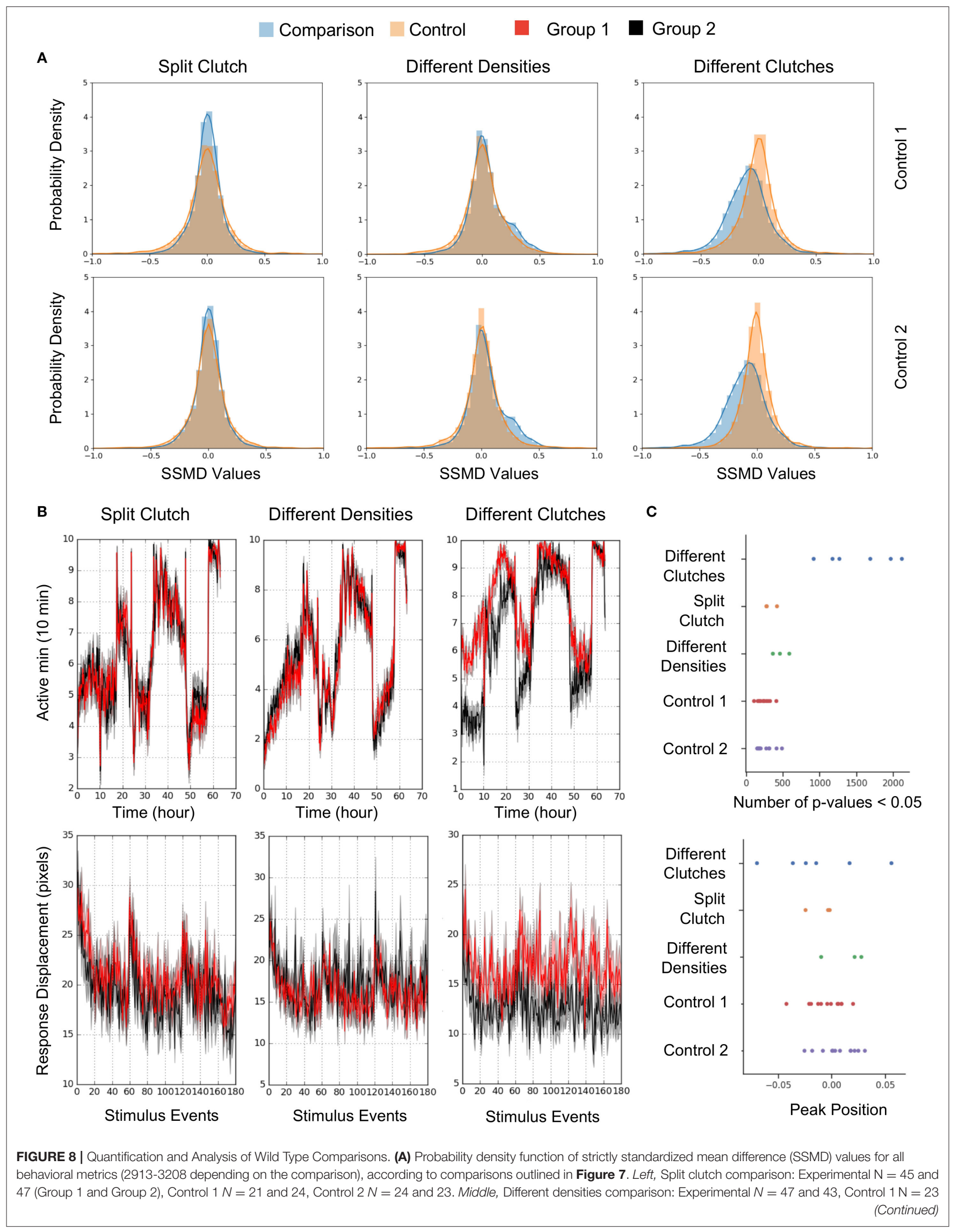


FIGURE 8 | and 23, Control $2 N=21$ and 23. Right, Different clutches comparison: Experimental $N=47$ and 46, Control $1 \mathrm{~N}=23$ and 24, Control $2 \mathrm{~N}=23$ and 23. (B) Example graphs for two measures included in Figure 7A: movement frequency and dark flash response displacement. Split clutch comparison: Kruskal-Wallis $p$-value for the Movement Frequency (Active minutes) metric $=0.751$. Kruskal-Wallis $p$-value for the dark flash Stimulus metric (Response Displacement) $=0.196$. Different densities comparison: Kruskal-Wallis $p$-value for the Movement Frequency (Active minutes) metric $=0.415$. Kruskal-Wallis $p$-value for the dark flash Stimulus metric (Response Displacement) $=0.993$. Different clutches comparison: Kruskal-Wallis $p$-value for the Movement Frequency (Active minutes) metric $=9.04 \times 10^{-9}$. Kruskal-Wallis $p$-value for the dark flash Stimulus metric (Response Displacement) $=6.05 \times 10^{-8}$. (C) Number of $p$-values $<0.05$ and the peak position of the kernel density estimation (KDE) curve for each comparison (Figure 8A). Twelve sets of comparisons with respective controls (Different clutch comparison: Four independent comparisons and two replicates, split clutch comparison: Three independent comparisons, different density comparison: Three independent comparisons). The total number of $p$-values ranged from 2,947 to 3,223 depending on the comparison.

interspersed PPI and control stimuli were separated by 3 min. Atxn7 (Figures 6A,B) and sbno1 (Figures 6C,D) mutants exhibited increased response frequency and magnitude relative to sibling controls with $1,000 \mathrm{~Hz}$ stimuli but not with $1,400 \mathrm{~Hz}$ stimuli. These results indicate that PPI phenotypes can vary according to acoustic stimulation frequency.

\section{Wild Type Comparisons}

While commonly used wild-type zebrafish strains exhibit substantial genetic diversity (Guryev et al., 2006; Brown et al., 2012), few studies explicitly define optimal growth and husbandry conditions that minimize possibly resultant behavioral variability.

As a first step to defining important parameters, we assessed three different conditions on larval zebrafish behavior. (1) To compare separately reared larvae, we divided sibling larvae into two dishes at identical density (Figure 7A). (2) To assess effects of density, we compared sibling larvae reared in two dishes of high or low density. (3) To compare nonsiblings, we raised different clutches at identical densities. For each experiment, we also compared within each experimental group as a control and interleaved animals from each condition in the 96-well plate to minimize possible positional effects (Figure 7B).

To estimate behavioral differences, we calculated strictly standardized mean difference (SSMD) values across all behavioral parameters (SSMD of 0 indicates no effect). Growing larvae in separate dishes or at different densities did not affect behavior, as demonstrated by largely overlapping SSMD distributions. However, larvae from different clutches exhibited significantly divergent SSMD distributions relative to control within-clutch comparisons (Figure 8A). For example, nonsibling larvae exhibited significantly different spontaneous movement frequency and dark flash response displacement, in contrast to siblings raised at identical or different densities (Figure 8B). Replicates of non-sibling comparisons generated greater numbers of $p$-values $<0.05$ and more divergent kernel density estimate peaks relative to other comparisons (Figure 8C). These results highlight the importance of comparing behavioral phenotypes within the same clutch.

\section{DISCUSSION}

The setup described above can test diverse zebrafish behaviors at high throughput and with minimal equipment and cost. In addition to baseline motion parameters, the system can assay prepulse inhibition (Figure 6) and responses to acoustic, visual, and thermal stimuli (Figure 5 and data not shown). We also incorporate a mini-projector to test additional visual behaviors such as the optomotor response (Figure 4). Together with different multi-well formats (such as 6-well plates with larger wells), our setup can accommodate more sophisticated visual assays including looming stimuli (Temizer et al., 2015; Marques et al., 2018), approach-avoidance to prey-predator (Barker and Baier, 2015), and decision-making based on dot coherence (Bahl and Engert, 2020). Because many components are commercially available, multiple boxes can be completely assembled within 2 days.

Our modular hardware design supports rapid adaptation for additional assays with adult animals or arena shapes beyond 96-well plate format. Modified camera/lens configurations can produce different resolutions or acquisition speeds. The circuit board design includes multiple BNC connectors capable of triggering or sampling from other devices. For example, these connectors can support optogenetic experiments (Oikonomou et al., 2019) as in the DanioVision system, or deliver electric shocks for conditioning assays (Valente et al., 2012), as in the ZebraBox system. Limitations of the current setup include the inability to present visual cues laterally (Bianco et al., 2011; Semmelhack et al., 2014) due to projector positioning, and experiments that require high-resolution tail and eye segmentation, as the camera and/or lens would need to be upgraded. Additionally, the system currently includes a single camera and collecting three-dimensional data requires modification of the LabVIEW software (Macrì et al., 2017).

The software to execute and analyze experiments is also highly adaptable. Existing experiment events can be modified to yield new event types. For example, users can acquire extended movies (Command ID "2") of a desired length or frame-rate by customizing the event type. These slowspeed movies provide opportunities for a wide range of new analyses that move beyond centroid/motion data, such as machine learning approaches to uncover phenotypes. This approach would have particular utility when monitoring older animals with more complex behaviors than larvae (Cachat et al., 2010; Pérez-Escudero et al., 2014; Dreosti et al., 2015; Geng and Peterson, 2019). While our Python-based analyses measure motion parameters more comprehensively than any commercially available zebrafish analysis software, machine learning may distinguish additional classes of movements or responses. For example, we do not explicitly distinguish O-bends and C-bends from other movements, but used parameters such as motion velocity to separate responses. Indeed, our analysis pipeline (Figure 3 ) is currently based 
solely on motion quantification, but is also highly flexible. A large set of input options can be modified from default settings without coding (Supplementary Material Data Sheet 1). Furthermore, the code's object-oriented and modular style permits independent modification of parameters such as the output graph format. However, a limitation of our platform is that a LabVIEW license is required. Users without access to LabVIEW can adapt open-source triggering software to collect movies (Lopes et al., 2015; Štih et al., 2019) while still utilizing our construction and analysis guidelines.

Using this new system, we assessed best practices for raising zebrafish larvae for behavioral experiments (Figures 7, 8). First, we found no effect of splitting a clutch across two petri dishes. While we routinely removed all debris from dishes during growth (see Methods), different levels of cleanliness may still influence behavior. Second, we observed no behavioral differences between larvae grown at two different densities, although densities higher than 150/dish were not tested and may negatively impact growth. Third, and most critical, we found that wild-type animals from different clutches exhibited behavioral differences of similar magnitude to mutants with the strongest behavioral phenotypes of 165 mutants (Thyme et al., 2019) vs. their respective control siblings (Data Sheet 1: Supplementary Figure 20). These results underscore the importance of comparing results within single clutches. We postulate that inter-clutch differences may contribute to variability in other contexts such as calcium imaging, where data is often collected from many parental pairs.

The zebrafish model continues to increase in popularity (Teame et al., 2019), while recent advances in genome editing technologies lower experimental barriers for nontraditional models. Our adaptable behavioral setup can monitor any small aquatic organism, particularly in multiwell format, and can thus accelerate discovery along both of these avenues. While neuroscientists likely represent the majority of users, our system can also serve as a powerful diagnostic tool for the development and function of other organs such as muscle (Maves, 2014). Finally, genome sequencing continues to link large numbers of genes to human disease (Schizophrenia Working Group of the Psychiatric Genomics Consortium, 2014; Satterstrom et al., 2020). The high throughput approaches outlined here will be critical to establish connections between disease-associated genes and decipher their neurobiological functions.

\section{REFERENCES}

Bahl, A., and Engert, F. (2020). Neural circuits for evidence accumulation and decision making in larval zebrafish. Nat. Neurosci. 23, 94-102. doi: 10.1038/s41593-019-0534-9

Barker, A. J., and Baier, H. (2015). Sensorimotor decision making in the zebrafish tectum. Curr. Biol. 25, 2804-2814. doi: 10.1016/j.cub.2015.09.055

Bianco, I. H., Kampff, A. R., and Engert, F. (2011). Prey capture behavior evoked by simple visual stimuli in larval zebrafish. Front. Syst. Neurosci. 5:101. doi: 10.3389/fnsys.2011.00101

\section{DATA AVAILABILITY STATEMENT}

The raw data supporting the conclusions of this article will be made available by the authors, without undue reservation.

\section{ETHICS STATEMENT}

The animal study was reviewed and approved by UAB Institutional Animal Care and Use Committee; Birmingham, Alabama.

\section{AUTHOR CONTRIBUTIONS}

WJ, BG, ES, and ST contributed to design and construction of the behavior setup. ST, WJ, and MV wrote the manuscript. ES built the majority of the LabVIEW software with contributions from MV and ST. ST built the majority of the Python software with contributions from MV. BG built the Arduino interface. ST, WJ, and MV conducted the experiments to demonstrate box functionality. All authors contributed to the article and approved the submitted version.

\section{FUNDING}

This research was supported by R00 MH110603 (ST), UL1TR003096 (UAB CCTS support to ST), and the UAB VSRC core grant P30 EY003039 for use of the supported electronics and machining services.

\section{ACKNOWLEDGMENTS}

The authors thank Verdion Martina, Gretchen Kioschos, and Emma Jones for assistance in building behavior boxes, Verdion Martina for assisting with data collection, Emma Jones for helpful comments on figures, Ari Ginsparg for assisting with Python environments, the UAB fish facility staff for zebrafish care, the UAB research computing team for providing and maintaining the Cheaha cluster, and the UAB electronics and machine shop. This manuscript has been released as a pre-print at BioRxiv (Joo et al., 2020).

\section{SUPPLEMENTARY MATERIAL}

The Supplementary Material for this article can be found online at: https://www.frontiersin.org/articles/10.3389/fnbeh. 2020.606900/full\#supplementary-material

Bolton, A. D., Haesemeyer, M., Jordi, J., Schaechtle, U., Saad, F. A., Mansinghka, V. K., et al. (2019). Elements of a stochastic 3D prediction engine in larval zebrafish prey capture. eLife 8:e51975. doi: 10.7554/eLife.519 75.sa2

Brown, K. H., Dobrinski, K. P., Lee, A. S., Gokcumen, O., Mills, R. E., Shi, X., et al. (2012). Extensive genetic diversity and substructuring among zebrafish strains revealed through copy number variant analysis. Proc. Natl. Acad. Sci. U.S.A. 109, 529-534. doi: 10.1073/pnas.1112163109

Burgess, H. A., and Granato, M. (2007). Sensorimotor gating in larval zebrafish. J. Neurosci. 27, 4984-4994. doi: 10.1523/JNEUROSCI.0615-07.2007 
Cachat, J., Stewart, A., Grossman, L., Gaikwad, S., Kadri, F., Chung, K. M., et al. (2010). Measuring behavioral and endocrine responses to novelty stress in adult zebrafish. Nat. Protoc. 5, 1786-1799. doi: 10.1038/nprot.2010.140

Chen, A., Singh, C., Oikonomou, G., and Prober, D. A. (2017). Genetic analysis of histamine signaling in larval zebrafish sleep. eNeuro 4:ENEURO.0286-16.2017. doi: 10.1523/ENEURO.0286-16.2017

Chiu, C. N., Rihel, J., Lee, D. A., Singh, C., Mosser, E. A., Chen, S., et al. (2016). A zebrafish genetic screen identifies neuromedin $U$ as a regulator of sleep/wake states. Neuron 89, 842-856. doi: 10.1016/j.neuron.2016.01.007

Dreosti, E., Lopes, G., Kampff, A. R., and Wilson, S. W. (2015). Development of social behavior in young zebrafish. Front. Neural Circuits 9:39. doi: 10.3389/fncir.2015.00039

Geng, Y., and Peterson, R. T. (2019). The zebrafish subcortical social brain as a model for studying social behavior disorders. Dis. Model. Mech. 12:dmm039446. doi: 10.1242/dmm.039446

Girard, S. L., Dion, P. A., Bourassa, C. V., Geoffroy, S., Lachance-Touchette, P., Barhdadi, A., et al. (2015). Mutation burden of rare variants in schizophrenia candidate genes. PLoS ONE 10:e0128988. doi: 10.1371/journal.pone.0128988

Griffin, A., Anvar, M., Hamling, K., and Baraban, S. C. (2020). Phenotype-based screening of synthetic cannabinoids in a dravet syndrome zebrafish model. Front. Pharmacol. 11:464. doi: 10.3389/fphar.2020.00464

Guryev, V., Koudijs, M. J., Berezikov, E., Johnson, S. L., Plasterk, R. H. A., van Eeden, F. J. M., et al. (2006). Genetic variation in the zebrafish. Genome Res. 16, 491-497. doi: 10.1101/gr.4791006

Horstick, E. J., Bayleyen, Y., and Burgess, H. A. (2020). Molecular and cellular determinants of motor asymmetry in zebrafish. Nat. Commun. 11:1170. doi: 10.1038/s41467-020-14965-y

Joo, W., Vivian, M.D., Graham, B.J., Soucy, E.R., and Thyme, S.B. (2020). A customizable low-cost system for massively parallel zebrafish behavior phenotyping. BioRxiv [preprint]. doi: 10.3389/fnbeh.2020.606900

Jordi, J., Guggiana-Nilo, D., Bolton, A. D., Prabha, S., Ballotti, K., Herrera, K., et al. (2018). High-throughput screening for selective appetite modulators: A multibehavioral and translational drug discovery strategy. Sci. Adv. 4:eaav1966. doi: 10.1126/sciadv.aav1966

Kokel, D., Bryan, J., Laggner, C., White, R., Cheung, C. Y. J., Mateus, R., et al. (2010). Rapid behavior-based identification of neuroactive small molecules in the zebrafish. Nat. Chem. Biol. 6, 231-237. doi: 10.1038/nchembio.307

Lee, D. A., Andreev, A., Truong, T. V., Chen, A., Hill, A. J., Oikonomou, G., et al. (2017). Genetic and neuronal regulation of sleep by neuropeptide VF. eLife 6:e25727. doi: 10.7554/eLife.25727.028

Leung, L. C., and Mourrain, P. (2016). Drug discovery: zebrafish uncover novel antipsychotics. Nat. Chem. Biol. 12, 468-469. doi: 10.1038/nchembio.2114

Lopes, G., Bonacchi, N., Frazão, J., Neto, J. P., Atallah, B. V., Soares, S., et al. (2015). Bonsai: an event-based framework for processing and controlling data streams. Front Neuroinformatics 9:7. doi: 10.3389/fninf.2015.00007

MacRae, C. A., and Peterson, R. T. (2015). Zebrafish as tools for drug discovery. Nat. Rev. Drug Discov. 14, 721-731. doi: 10.1038/nrd4627

Macrì, S., Neri, D., Ruberto, T., Mwaffo, V., Butail, S., and Porfiri, M. (2017). Three-dimensional scoring of zebrafish behavior unveils biological phenomena hidden by two-dimensional analyses. Sci. Rep. 7:1962. doi: 10.1038/s41598-017-01990-z

Marques, J. C., Lackner, S., Félix, R., and Orger, M. B. (2018). Structure of the zebrafish locomotor repertoire revealed with unsupervised behavioral clustering. Curr. Biol. 28, 181-195.e5. doi: 10.1016/j.cub.2017. 12.002

Maves, L. (2014). Recent advances using zebrafish animal models for muscle disease drug discovery. Expert Opin. Drug Discov. 9, 1033-1045. doi: 10.1517/17460441.2014.927435

Naumann, E. A., Fitzgerald, J. E., Dunn, T. W., Rihel, J., Sompolinsky, H., and Engert, F. (2016). From whole-brain data to functional circuit models: the zebrafish optomotor response. Cell 167, 947-960.e20. doi: 10.1016/j.cell.2016.10.019
Oikonomou, G., Altermatt, M., Zhang, R.-W., Coughlin, G. M., Montz, C., Gradinaru, V., et al. (2019). The serotonergic raphe promote sleep in zebrafish and mice. Neuron 103, 686-701.e8. doi: 10.1016/j.neuron.2019.05.038

Pérez-Escudero, A., Vicente-Page, J., Hinz, R. C., Arganda, S., and de Polavieja, G. G. (2014). idTracker: tracking individuals in a group by automatic identification of unmarked animals. Nat. Methods 11, 743-748. doi: $10.1038 /$ nmeth.2994

Randlett, O., Haesemeyer, M., Forkin, G., Shoenhard, H., Schier, A. F., Engert, F., et al. (2019). Distributed plasticity drives visual habituation learning in larval zebrafish. Curr. Biol. 29, 1337-1345.e4. doi: 10.1016/j.cub.2019.02.039

Rihel, J., Prober, D. A., Arvanites, A., Lam, K., Zimmerman, S., Jang, S., et al. (2010). Zebrafish behavioral profiling links drugs to biological targets and rest/wake regulation. Science 327, 348-351. doi: 10.1126/science.1183090

Satterstrom, F. K., Kosmicki, J. A., Wang, J., Breen, M. S., De Rubeis, S., An, J.-Y., et al. (2020). Large-scale exome sequencing study implicates both developmental and functional changes in the neurobiology of autism. Cell 180, 568-584.e23. doi: 10.1016/j.cell.2019.12.036

Schizophrenia Working Group of the Psychiatric Genomics Consortium (2014). Biological insights from 108 schizophrenia-associated genetic loci. Nature 511, 421-427. doi: 10.1038/nature13595

Semmelhack, J. L., Donovan, J. C., Thiele, T. R., Kuehn, E., Laurell, E., and Baier, H. (2014). A dedicated visual pathway for prey detection in larval zebrafish. eLife 3:e04878. doi: 10.7554/eLife.04878.018

Singh, C., Rihel, J., and Prober, D. A. (2017). Neuropeptide Y regulates sleep by modulating noradrenergic signaling. Curr. Biol. 27, 3796-3811.e5. doi: 10.1016/j.cub.2017.11.018

Štih, V., Petrucco, L., Kist, A. M., and Portugues, R. (2019). Stytra: An open-source, integrated system for stimulation, tracking and closed-loop behavioral experiments. PLoS Comput. Biol. 15:e1006699. doi: 10.1371/journal.pcbi.1006699

Teame, T., Zhang, Z., Ran, C., Zhang, H., Yang, Y., Ding, Q., et al. (2019). The use of zebrafish (Danio rerio) as biomedical models. Anim. Front. 9, 68-77. doi: 10.1093/af/vfz020

Temizer, I., Donovan, J. C., Baier, H., and Semmelhack, J. L. (2015). A visual pathway for looming-evoked escape in larval zebrafish. Curr. Biol. 25, 1823-1834. doi: 10.1016/j.cub.2015.06.002

Thyme, S. B., Pieper, L. M., Li, E. H., Pandey, S., Wang, Y., Morris, N. S., et al. (2019). Phenotypic landscape of schizophrenia-associated genes defines candidates and their shared functions. Cell 177, 478-491.e20. doi: 10.1016/j.cell.2019.01.048

Valente, A., Huang, K.-H., Portugues, R., and Engert, F. (2012). Ontogeny of classical and operant learning behaviors in zebrafish. Learn. Mem. 19, 170-177. doi: 10.1101/lm.025668.112

Wolman, M. A., Jain, R. A., Liss, L., and Granato, M. (2011). Chemical modulation of memory formation in larval zebrafish. Proc. Natl. Acad. Sci. U.S.A. 108, 15468-15473. doi: 10.1073/pnas.1107156108

Woods, I. G., Schoppik, D., Shi, V. J., Zimmerman, S., Coleman, H. A., Greenwood, J., et al. (2014). Neuropeptidergic signaling partitions arousal behaviors in zebrafish. J. Neurosci. 34, 3142-3160. doi: 10.1523/JNEUROSCI.3529-13.2014

Conflict of Interest: The authors declare that the research was conducted in the absence of any commercial or financial relationships that could be construed as a potential conflict of interest.

Copyright (c) 2021 Joo, Vivian, Graham, Soucy and Thyme. This is an open-access article distributed under the terms of the Creative Commons Attribution License (CC $B Y)$. The use, distribution or reproduction in other forums is permitted, provided the original author(s) and the copyright owner(s) are credited and that the original publication in this journal is cited, in accordance with accepted academic practice. No use, distribution or reproduction is permitted which does not comply with these terms. 